\title{
Effects of genome-wide copy number variation on expression in mammalian cells
}

\author{
Richard T Wang ${ }^{1}$, Sangtae Ahn ${ }^{2,5}$, Christopher C Park', Arshad H Khan ${ }^{1}$, Kenneth Lange ${ }^{3,4}$ and Desmond J Smith ${ }^{\text {* }}$
}

\begin{abstract}
Background: There is only a limited understanding of the relation between copy number and expression for mammalian genes. We fine mapped cis and trans regulatory loci due to copy number change for essentially all genes using a human-hamster radiation hybrid (RH) panel. These loci are called copy number expression quantitative trait loci (ceQTLs).
\end{abstract}

Results: Unexpected findings from a previous study of a mouse-hamster RH panel were replicated. These findings included decreased expression as a result of increased copy number for 30\% of genes and an attenuated relationship between expression and copy number on the $X$ chromosome suggesting an Xist independent form of dosage compensation. In a separate glioblastoma dataset, we found conservation of genes in which dosage was negatively correlated with gene expression. These genes were enriched in signaling and receptor activities. The observation of attenuated X-linked gene expression in response to increased gene number was also replicated in the glioblastoma dataset. Of 523 gene deserts of size $>600 \mathrm{~kb}$ in the human RH panel, 325 contained trans ceQTLs with $-\log _{10} P>4.1$. Recently discovered genes, ultra conserved regions, noncoding RNAs and microRNAs explained only a small fraction of the results, suggesting a substantial portion of gene deserts harbor as yet unidentified functional elements.

Conclusion: Radiation hybrids are a useful tool for high resolution mapping of cis and trans loci capable of affecting gene expression due to copy number change. Analysis of two independent radiation hybrid panels show agreement in their findings and may serve as a discovery source for novel regulatory loci in noncoding regions of the genome.

\section{Background}

Radiation hybrid $(\mathrm{RH})$ panels were originally devised to build high resolution maps of mammalian genomes $[1,2]$. The panels are created by lethally irradiating a donor cell (mouse, human, rat, etc) harboring a selectable marker and propagating the resulting DNA fragments by fusing the donor cells with the recipient hamster cell line A23. Each clone in an RH panel contains a random assortment of the donor DNA permitting construction of a physical map. Since high doses of radiation can be used, a large number of breakpoints can be obtained, $>10^{4}$ in a typical panel of $\sim 100$ clones.

$\mathrm{RH}$ panels exhibit copy number variation (CNV) for essentially all genes and represent a powerful resource

\footnotetext{
* Correspondence: dsmith@mednet.ucla.edu

'Department of Molecular and Medical Pharmacology, David Geffen School of Medicine, University of California, Los Angeles, CA 90095, USA Full list of author information is available at the end of the article
}

for unbiased examination of CNV-induced effects on gene expression. The existence of CNVs across multiple clones in a panel boosts statistical power.

Studies that map quantitative trait loci (QTLs) regulating gene expression (expression QTLs or eQTLs) usually rely on naturally occurring polymorphisms as a source of genetic variation and meiotic recombination to narrow down the regulatory loci. Frequently, the mechanistic significance of naturally occurring polymorphisms in affecting gene expression is not immediately apparent from the context. Genetic alterations due to CNVs have recently come to the fore as a source of considerable polymorphism in humans $[3,4]$. In contrast to other polymorphisms, a one to one correspondence between copy number and gene expression is, on its face, a reasonable expectation for CNVs although exceptions have been noted [5-7]. Since the variation in RH cells is due to CNVs, we refer to loci affecting expression in the $\mathrm{RH}$ 
panels copy number eQTLs or ceQTLs. However, unlike naturally occurring CNVs, variation in the RH panels is uniform and genome-wide.

Recently, array comparative genomic hybridization $(\mathrm{aCGH})$ and gene expression microarrays were used to fine map loci regulating expression genome-wide in a mouse-hamster radiation hybrid panel [8]. The analysis of the mouse RH panel revealed a number of unexpected findings. These included the fact that $\sim 30 \%$ of genes showed decreased gene expression in response to increased copy number, a potentially novel form of dosage compensation for the $\times$ chromosome independent of $\times$ chromosome inactivation, and the existence of ceQTLs in noncoding regions of the genome.

To further investigate these surprising findings, we used the Stanford G3 radiation hybrid panel [9]. The 83 clones in this panel are derived from a human male donor genome. We also used publicly available glioblastoma multiforme (GBM) data from The Cancer Genome Atlas (TCGA). We found consistent overlap between the human $\mathrm{RH}$, mouse $\mathrm{RH}$ and TCGA data in terms of regulated pathways for genes with negative correlation between CNVs and expression data and attenuated response of X-linked genes in response to copy number increase. In addition, we found ceQTLs in non-genic regions in the two RH datasets and that these nongenic ceQTLs could not be explained by recently discovered exotic transcripts in noncoding regions harboring ceQTLs.

\section{Results}

\section{Gene expression}

RNA was extracted from each of the 79 available radiation hybrid clones and technical replicates hybridized to Illumina HumanRef-8 v1.0 BeadChips. The relative hybridization efficiencies of hamster and human transcripts on the arrays were comparable (Additional File 1 Figure S1A-D) and there was good reproducibility between duplicate arrays (Additional File 2 Figure S2).

\section{Assessing copy number and retention frequency in the G3 RH panel}

To measure DNA copy number in the RH cell lines, we used array comparative genomic hybridization (aCGH) of each clone compared to the reference hamster A23 recipient line. The aCGH genotyping agreed well with the historical PCR genotyping $\left(\chi^{2}=159,996,1\right.$ d.f., $P<$ $2.2 \times 10^{-16}$ ) (Additional File 3 Figure S3A). The average loss of PCR markers across all RH cells was $36.6 \%$. This loss is likely due to the multiple passages of the $\mathrm{RH}$ clones since its creation a decade ago. Almost no gain of markers $(<1 \%)$ was observed. Individual cell lines showed large variation in loss, ranging from 3-96\%. The final retention frequency (i.e., average amount of donor
DNA retained per $\mathrm{RH}$ clone) was $11.4 \%$. The average donor DNA fragment length was $4 \mathrm{Mb}$.

Across the 79 available clones in the G3 RH panel, the entire human genome is represented, on average, nine times $(0.11 \times 79=9)$ (Additional File 3 Figure S3B), although a few regions were extreme. As expected, the retention of the region surrounding thymidine kinase (Tk1), the selectable marker used in creating the panel was $100 \%$. Human centromeric regions were preferentially retained in the $\mathrm{RH}$ cell lines (Welch's $t$-test, $P<$ $10^{-15}$ ) (Additional File 3 Figure S3C-E), as found previously [9]. This observation implies that human centromeres function efficiently in hamster cells despite lineage differences [10].

\section{Gene expression changes with copy number}

We used a previously described linear regression model [8] to relate changes in gene expression to copy number in the 79 available clones (Methods). Briefly, the $\log _{10}$ ( $\mathrm{RH}$ expression/A23 control) of each gene served as the dependent variable while the $\log _{10}(\mathrm{RH} / \mathrm{A} 23) \mathrm{CGH}$ intensity served as the regressor. The change in expression due to copy number is characterized by an effect size parameter $\alpha$ (Figure 1A, B). If $\alpha=1$, gene expression is exactly proportional to copy number. The significance of $\alpha$ was assessed by permutation testing (Methods).

We defined a cis ceQTL as a locus within a $5 \mathrm{Mb}$ radius of a regulated gene (Figure $1 \mathrm{C}$ ). Trans ceQTLs were defined as loci regulating genes at a distance greater than $5 \mathrm{Mb}$ or on another chromosome (Figure 1D). To account for multiple hypothesis testing, we applied false discovery rates (FDRs) to cis and trans

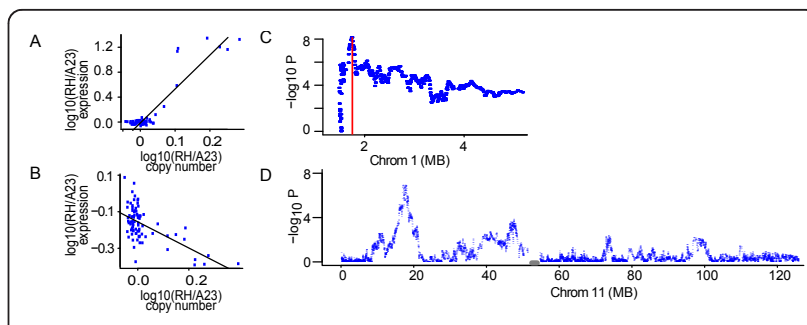

Figure 1 Cis and trans human ceQTLs. (A) Trans ceQTL. RPL18P4 on chromosome 7 displays an increase in $\log _{10}(\mathrm{RH} / \mathrm{A} 23)$ gene expression as $\log _{10}(\mathrm{RH} / \mathrm{A} 23)$ copy number of a marker at $53.7 \mathrm{Mb}$ on chromosome 19 increases $\left(\alpha=5.2,-\log _{10} P=8.35\right)$. (B) Example of negative $\alpha$ trans ceQTL. $\log _{10}(\mathrm{RH} / \mathrm{A} 23)$ gene expression of DPH3 on chromosome 3 decreases as $\log _{10}(\mathrm{RH} / \mathrm{A} 23)$ copy number of a marker at $128.1 \mathrm{Mb}$ on chromosome 12 increases $\left(\alpha=-0.72\right.$, $-\log _{10}$ $P=4.27$ ). (C) Cis ceQTL for GNB1 is located at $1.7 \mathrm{Mb}$ on the beginning portion of chromosome 1. Red line denotes position of regulated gene. (D) Multiple trans ceQTLs on Chromosome 11 regulating RPS13 on chromosome 1. The peaks located at 17.1 Mb and $47 \mathrm{Mb}$ show strongest evidence of regulation. Points are aCGH markers plotted along chromosomes. Centromeres are grey. 
ceQTLs separately $[11,12]$. We used an FDR threshold of $<0.25$ for our ceQTLs in the human data giving 15,263 cis ceQTLs. In mouse, the same threshold gave 16,234 cis ceQTLs. For trans ceQTLs, we also used FDR $<0.25$ in both mouse and human data. These FDRs correspond to $P$ values of 0.09 and $2.92 \times 10^{-5}$ for $c i s$ and trans ceQTLs, respectively in the human RH data (Figure $2 \mathrm{~A}-\mathrm{B})$.

Figure 3 shows the landscape of ceQTLs at various FDRs. Consistent with other eQTL mapping studies [13], we found trans bands which may indicate hotspots of regulatory activity (Figure 3 horizontal marginal). Genes regulated by multiple loci (Figure 3 vertical marginal) may represent key genes integrating multiple pathways. The high breakpoint density of the RH panel permitted multiple regulatory loci along individual chromosomes to be resolved (Figure 1D).

\section{Cis ceQTLs and genes that turn down their own expression}

The median distance between a gene and its cis ceQTL was $531 \mathrm{~kb}$ (Additional File 4 Figure S4A). Cis ceQTL effect sizes $(\alpha)$ showed a bimodal distribution with means of 0.73 and -0.12 for positive and negative $\alpha$ 's, respectively. (Additional File 4 Figure S4B). A total of 5,831 of 16,234 (36\%) cis ceQTLs decreased their gene expression when their copy number was increased (i.e., possessed negative $\alpha$ ). In our mouse RH, $30 \%$ of cis $\alpha$ were negative at $\mathrm{FDR}<0.25$.

After identifying $\sim 11,000$ orthologous genes between mouse and human, we determined the number of common genes whose expression correlated with copy number to be 7,936. Human and mouse possessed 6,092 and 5,979 genes whose expression increased with copy number respectively and 1,844 and 1,957 genes whose expression was inversely correlated with copy number respectively. 4,805 genes had positive $\alpha$ and 670 had negative $\alpha$ in both human and mouse data. A chi-square test showed enrichment of both positive cis $\alpha$ and negative cis $\alpha$ ceQTLs across both species $\left(P<2.2 \times 10^{-16}\right)$,

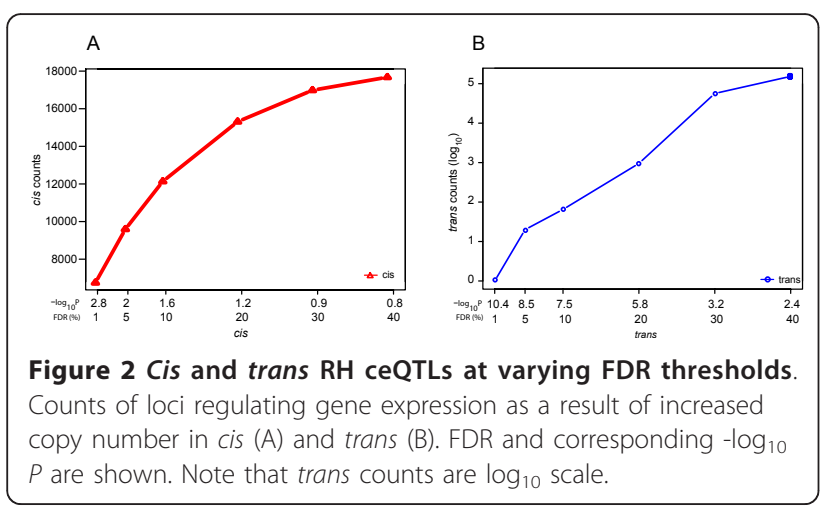

suggesting that negative cis $\alpha$ ceQTLs are not simply due to noise. Using a more stringent cutoff of FDR 5\% in the human RH data, 198 negative correlations still persist. In the mouse RH data at an FDR of 5\%, 172 negative correlations still exist and the overlap of 32 genes with negative $\alpha$ is statistically significant $\left(\mathrm{P}=1.04 \times 10^{-7}\right)$.

We employed DAVID [14] to search the Gene Ontology for functional enrichment of genes with negative cis $\alpha$ in the human and mouse RH datasets (Table 1). There was a high degree of functional conservation for genes with negative cis $\alpha$ with the most enriched categories involving membrane functions, receptor activity and signaling. Consistent with this observation, a Mann-Whitney rank test of the GO scores between the two data sets was significant $\left(\mathrm{P}=1.34 \times 10^{-7}\right)$. The trend was less clear among genes with positive cis $\alpha$ in the mouse and human (Additional file 5 Table S1) with categories such as metabolic process, regulation of apoptosis and binding highly conserved between the two species.

A recent study of cells trisomic for each of the mouse chromosomes 1, 13, 16 and 19 [15] provided an opportunity to test our negative cis $\alpha$ ceQTLs and further rule out noise as a cause of this surprising phenomenon. Similar to the analysis of the RH panels, we used linear regression to estimate effect sizes due to copy number increases in the aneuploid cells. Out of 1,699 orthologous genes between mouse RH and mouse trisomy data, 1,275 and 1,191 cis ceQTLs had positive cis $\alpha$ in the trisomy and mouse RH data respectively and 424 and 508 possessed negative cis $\alpha$ respectively. A chi-square test showed enrichment of both positive cis $\alpha$ and negative cis $\alpha$ ceQTLs $\left(P=7.4 \times 10^{-9}\right)$. We repeated this test using human RH and mouse aneuploidy data $(1,213$ orthologous genes) and found a highly significant overlap of 131 genes with negative cis $\alpha\left(P=8.2 \times 10^{-12}\right)$. The replicability of the negative $\alpha$ findings across these datasets argues in favor of a true biological phenomenon.

Absolute expression levels of genes with positive cis $\alpha$ is statistically significant from genes with negative cis $\alpha$ $\left(\mathrm{P}=4.5 \times 10^{-9}\right)$, although this difference is due to a fraction of highly expressed genes with positive cis $\alpha$ (Additional File 6 Figure S5A). The mean gene expression values were quite close (12.04 versus 11.99 , positive and negative $\alpha$ respectively). Cis ceQTLs with negative alpha show little evidence of antisense transcription ( 289 out of 5,831$)$ and the genes underlying them were largely found in their entirety across all $79 \mathrm{RH}$ cell lines (Additional File 6 Figure S5B). In addition, neighboring markers nearly always had concordant $\alpha$ (Additional File 6 Figure S5C-D).

\section{Decreased cis effects on $\mathrm{X}$ chromosome}

Genes on the X chromosome in the human RH dataset showed a significantly attenuated cis response to 


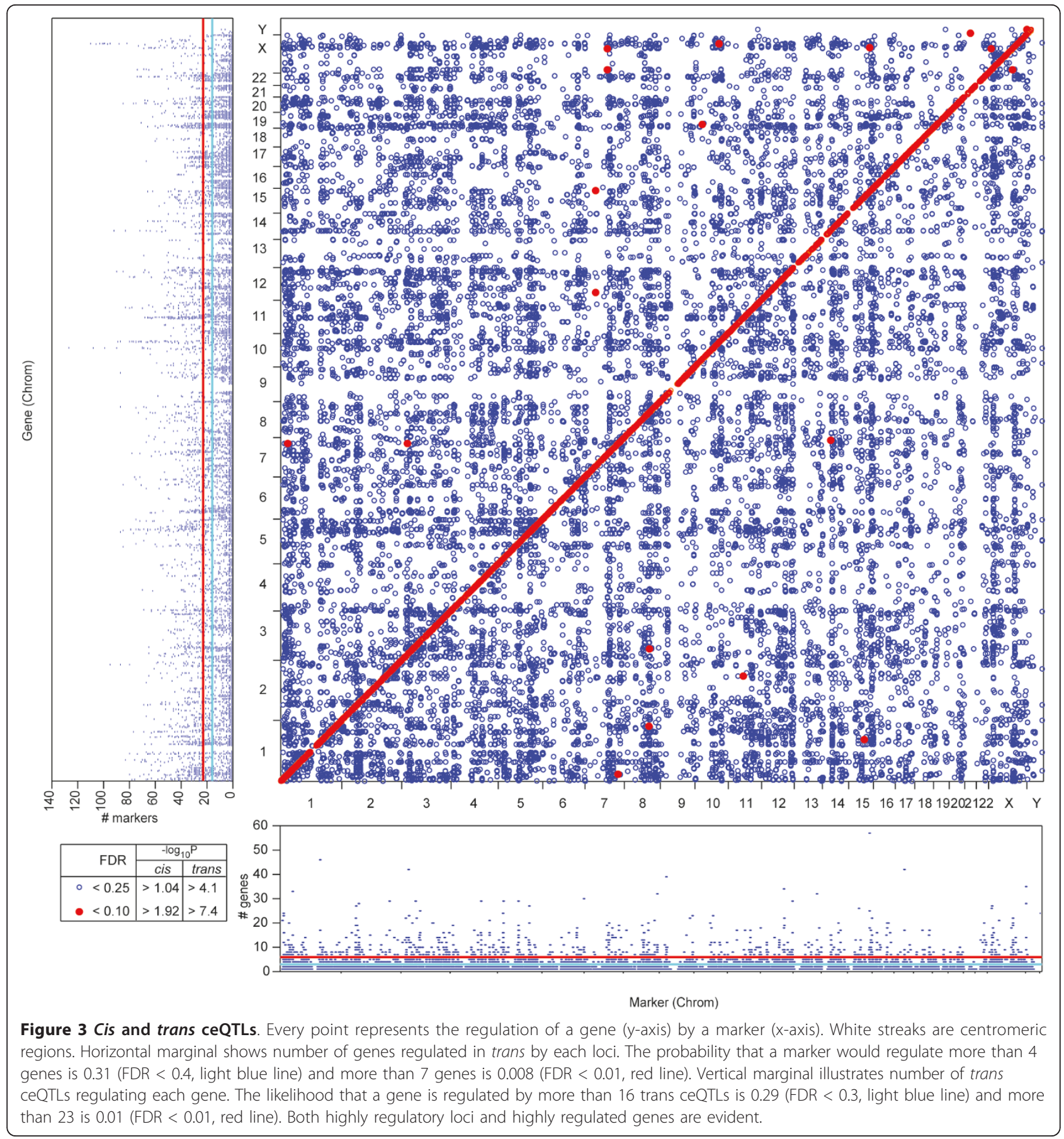

increased copy number compared to the autosomes (Figure 4A). The same phenomenon was also found in our mouse dataset, where the mean cis $\alpha$ on the $\mathrm{X}$ chromosome was significantly less (roughly half) than the autosomes [8] (Figure 4B). We performed a paired t-test by evaluating the average positive cis $\alpha$ for autosomes and $\mathrm{X}$ chromosomes for each human $\mathrm{RH}$ clone and found a significant difference $\left(P=6.7 \times 10^{-9}, 78\right.$ d.f. $)$ between the two. The same was true for negative cis $\alpha$
$\left(P=1.9 \times 10^{-13}, 78\right.$ d.f.) (Figure 4D). Both the human donor and A23 hamster cells used to construct the hybrids are male. Because donor chromosomes are fragmented by irradiation and concatenated to other random fragments upon incorporation into the recipient cell line, possible activation of the donor Xist locus would not consistently silence all X chromosome genes. Since the retention frequency of the donor X chromosome is only 5.9\% (Additional File 3 Figure S3F), 
Table 1 GO Enrichment for negative cis $\alpha$ at FDR $<0.25$

\begin{tabular}{|c|c|c|c|c|c|}
\hline Human & Score & FDR & Mouse & Score & FDR \\
\hline \multicolumn{6}{|l|}{ Biological Process } \\
\hline System Development & $5.55 \times 10^{-38}$ & $8.77 \times 10^{-35}$ & System Development & $2.22 \times 10^{-19}$ & $3.45 \times 10^{-16}$ \\
\hline Cell-Cell Signaling & $1.57 \times 10^{-36}$ & $2.49 \times 10^{-33}$ & Organ Development & $1.08 \times 10^{-15}$ & $1.73 \times 10^{-12}$ \\
\hline Cell Differentiation & $1.88 \times 10^{-24}$ & $2.97 \times 10^{-21}$ & Cell Differentiation & $4.86 \times 10^{-14}$ & $7.57 \times 10^{-11}$ \\
\hline Ion Transport & $3.11 \times 10^{-24}$ & $4.93 \times 10^{-21}$ & Cell-Cell Signaling & $6.67 \times 10^{-12}$ & $1.04 \times 10^{-8}$ \\
\hline Organ Development & $1.06 \times 10^{-23}$ & $1.68 \times 10^{-20}$ & Positive Regulation of Biological Process & $2.94 \times 10^{-10}$ & $4.58 \times 10^{-7}$ \\
\hline \multicolumn{6}{|l|}{ Cellular Component } \\
\hline Plasma Membrane Part & $2.38 \times 10^{-53}$ & $3.53 \times 10^{-50}$ & Plasma Membrane & $1.60 \times 10^{-27}$ & $2.30 \times 10^{-24}$ \\
\hline Plasma Membrane & $3.62 \times 10^{-46}$ & $5.37 \times 10^{-43}$ & Extracellular Region & $1.04 \times 10^{-15}$ & $1.43 \times 10^{-12}$ \\
\hline Intrinsic To Plasma Membrane & $5.56 \times 10^{-45}$ & $8.26 \times 10^{-42}$ & Plasma Membrane Part & $9.65 \times 10^{-14}$ & $1.38 \times 10^{-10}$ \\
\hline Integral To Plasma Membrane & $9.54 \times 10^{-44}$ & $1.42 \times 10^{-40}$ & Synapse Part & $4.67 \times 10^{-12}$ & $6.70 \times 10^{-9}$ \\
\hline \multicolumn{6}{|l|}{ Molecular Function } \\
\hline $\begin{array}{l}\text { Passive Transmembrane Transporter } \\
\text { Activity }\end{array}$ & $2.87 \times 10^{-27}$ & $3.75 \times 10^{-24}$ & $\begin{array}{l}\text { Passive Transmembrane Transporter } \\
\text { Activity }\end{array}$ & $2.06 \times 10^{-11}$ & $2.56 \times 10^{-8}$ \\
\hline $\begin{array}{l}\text { Substrate-Specific Transmembrane } \\
\text { Transporter Activity }\end{array}$ & $3.85 \times 10^{-18}$ & $5.03 \times 10^{-15}$ & $\begin{array}{l}\text { Substrate-Specific Transmembrane } \\
\text { Transporter Activity }\end{array}$ & $4.49 \times 10^{-7}$ & $5.59 \times 10^{-4}$ \\
\hline Receptor Binding & $1.55 \times 10^{-13}$ & $2.03 \times 10^{-10}$ & Receptor Binding & $2.05 \times 10^{-5}$ & $2.54 \times 10^{-2}$ \\
\hline Receptor Activity & $9.80 \times 10^{-13}$ & $1.28 \times 10^{-9}$ & Heme Binding & $1.07 \times 10^{-4}$ & $1.3 \times 10^{-1}$ \\
\hline
\end{tabular}

activation of the recipient hamster Xist locus would render the RH clones functionally haploid for most of the $\mathrm{X}$ chromosome and would be inviable. Thus the attenuated cis ceQTL effect size on the X chromosome implies a Xist-independent dosage compensation mechanism for $\mathrm{X}$-linked genes.

\section{Cis ceQTLs in cancer and RH cells have similar properties}

Using glioblastoma multiforme (GBM) cancer data publicly available from the Cancer Genome Atlas (TCGA) project, we applied linear regression to estimate cis copy number effects on gene expression and then compared the results to our human and mouse RH panels. While not a perfect analogue to RH panels, cancer often possess alterations in copy number which would be expected to influence gene expression. In the cancer data, $38.7 \%$ of the human genome showed copy number variation. X chromosomal coverage was $68.8 \%$. Similar to the $\mathrm{RH}$ analysis, we employed a $5 \mathrm{Mb}$ radius for cis effects and corrected $P$ values such that FDR $<0.05$ ( $P$ $<0.04)$.

At FDR $<0.05,8,764$ genes in the TCGA dataset showed cis effects between copy number and gene expression of which 5,815 were common to the human RH data. Human RH and TCGA had 4,670 and 5,320 cis ceQTLs with positive $\alpha$ and 1,145 and 495 cis ceQTLs with negative $\alpha$. Enrichment of cis ceQTLs that had positive and negative $\alpha$ in both data sets (4,372 and 197 for positive and negative $\alpha$ respectively) was demonstrated by chi-square $\left(P<2.2 \times 10^{-16}\right)$. The same was true for TCGA and mouse RH $\left(P=2.5 \times 10^{-11}\right)$. The number of genes with negative correlation between copy number and gene expression in common between the TCGA, human RH and mouse RH datasets is 42 (Additional File 7 Table S2). Despite the modest number of overlapping genes, the GO categories for the 720 genes with negative correlation between copy number and expression in the cancer data were strikingly similar to the RH genes with negative $\alpha$ and included categories of plasma membrane, signaling and receptor activity (Table 2). A Mann-Whitney rank test between TCGA and human RH GO scores was significant $\left(P=3.2 \times 10^{-6}\right)$.

We sought confirmation of decreased cis effects on the $\mathrm{X}$ chromosome in TCGA data. We used male TCGA samples $(\mathrm{N}=180)$ to exclude the effects of $\mathrm{X}$ chromosome inactivation (Figure 4C). However, similar conclusions were drawn from female TCGA data ( $\mathrm{N}=52$, Figure 4D). In relation to the autosomes, mean gene expression on the X chromosome in the male TCGA samples showed a significant attenuation in response to increased copy number (Figure 4C). We divided X-linked and autosomal genes in the TCGA data into positively and negatively regulating cis ceQTLs and found that genes on the $\mathrm{X}$ chromosome possessed smaller effect sizes than the autosomes (paired t-test, 179 d.f., $P<2.2 \times 10^{-16}$ for both positive and negative). This is similar to what we observed in both human and mouse $\mathrm{RH}$ panels where effect sizes for genes on $\mathrm{X}$ were smaller in magnitude than autosomes (Figure 4D). Considering the selective pressure in cancer cells and the corresponding lack of uniform coverage compared to RH cells, overall, TCGA data is consistent with the findings of negative cis 


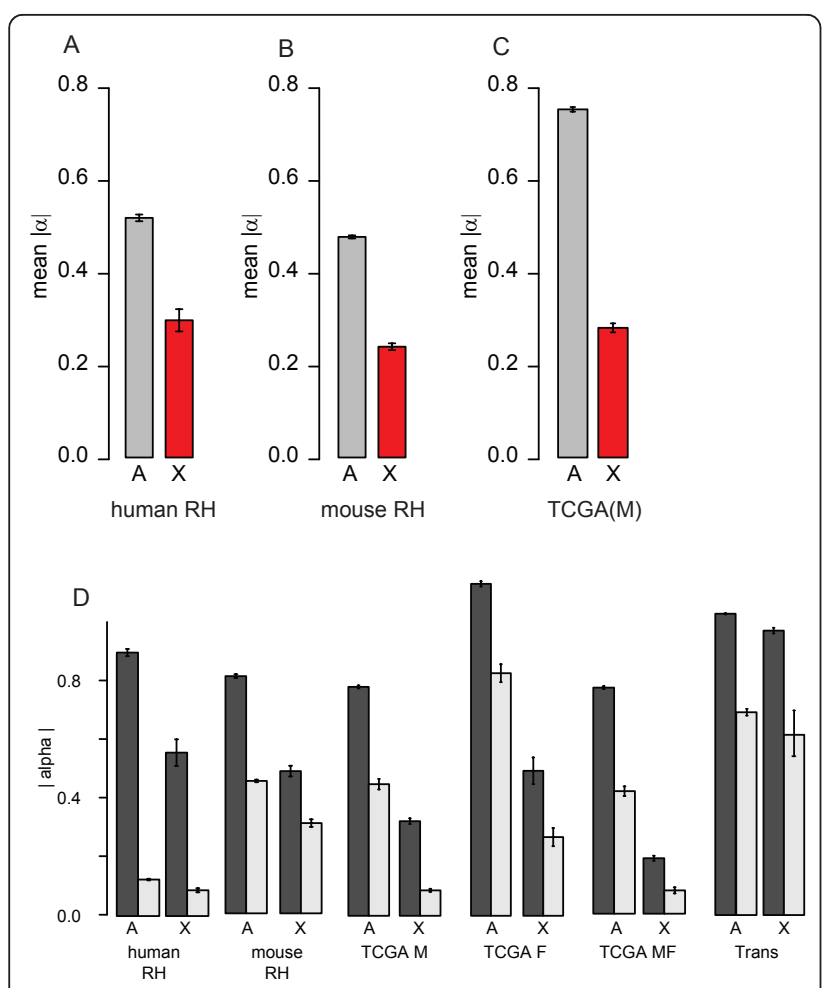

Figure 4 Cis ceQTLs on autosomes versus $\mathbf{X}$ chromosome. Mean absolute value cis $\alpha$ on autosomes and $X$ chromosomes for (A) human $\mathrm{RH}$, (B) mouse $\mathrm{RH}$ and (C) TCGA male. (D) Mean absolute value cis $\alpha$ for positive (dark grey) and negative (light grey) ceQTLs on the autosomes and $X$ chromosome in human $\mathrm{RH}$, mouse $\mathrm{RH}$, male TCGA, female TCGA and all TCGA. Mean absolute value $\alpha$ for $X$-linked genes regulated in trans are also shown. Error bars are s.e. $\mathrm{m}$

ceQTLs and the attenuated X-linked copy number/ expression relationships in the RH datasets.

\section{Trans ceQTLs}

There were a total of 17,347 trans loci at an FDR $<0.25$. Of the 36,082 trans interactions between peak markers and genes in the human RH data, 39 have negative $\alpha$ (indicating repression) while the remaining 36,043 (99.9\%) have positive $\alpha$ (induction).

Both the mouse and human $\mathrm{RH}$ datasets had genes regulated by multiple loci (Figure 3 horizontal marginal). To test for conservation of hotspots regulating multiple genes in trans (Figure 3 vertical marginal) in human and mouse, we remapped mouse ceQTLs onto the human genome using the UCSC Liftover utility. We then binned the human genome into $1 \mathrm{Mb}$ bins and performed a chi-square test on the number of genes regulated by each bin in the two $\mathrm{RH}$ datasets. The result was not significant.

We then investigated the overlap of genes underlying trans ceQTLs between human and mouse RH data. For
Table 2 GO Enrichment for negative cis $\alpha$ in TCGA

\begin{tabular}{lll}
\hline Human & Score & FDR \\
\hline Biological Process & & \\
\hline Multicellular Organismal Process & $1.05 \times 10^{-12}$ & $1.90 \times 10^{-9}$ \\
\hline Cell-Cell Signaling & $9.28 \times 10^{-11}$ & $1.67 \times 1^{-7}$ \\
\hline Cell Communication & $4.33 \times 10^{-10}$ & $7.81 \times 1^{-7}$ \\
\hline Immune Response & $6.04 \times 10^{-10}$ & $1.09 \times 10^{-6}$ \\
\hline Immune System Process & $1.35 \times 10^{-9}$ & $2.44 \times 10^{-6}$ \\
\hline Response To Stimulus & $5.88 \times 10^{-9}$ & $1.06 \times 10^{-5}$ \\
\hline Anatomical Structure Development & $1.14 \times 10^{-8}$ & $2.06 \times 10^{-5}$ \\
\hline System Development & $2.14 \times 10^{-8}$ & $3.85 \times 10^{-5}$ \\
\hline Organ Development & $5.01 \times 10^{-8}$ & $9.04 \times 10^{-5}$ \\
\hline System Process & $6.70 \times 10^{-8}$ & $1.21 \times 10^{-4}$ \\
\hline Transmission Of Nerve Impulse & $1.32 \times 10^{-7}$ & $2.39 \times 10^{-4}$ \\
\hline Defense Response & $1.72 \times 10^{-7}$ & $3.10 \times 10^{-4}$ \\
\hline Multicellular Organismal Development & $2.42 \times 10^{-7}$ & $4.37 \times 10^{-4}$ \\
\hline Developmental Process & $5.27 \times 10^{-7}$ & $9.50 \times 10^{-4}$ \\
\hline Signal Transduction & $9.39 \times 10^{-7}$ & $1.69 \times 10^{-3}$ \\
\hline Cellular Component & & \\
\hline Intrinsic To Plasma Membrane & $3.97 \times 10^{-22}$ & $5.55 \times 10^{-19}$ \\
\hline Integral To Plasma Membrane & $5.78 \times 10^{-22}$ & $8.08 \times 10^{-19}$ \\
\hline Plasma Membrane & $7.84 \times 10^{-20}$ & $1.10 \times 10^{-16}$ \\
\hline Plasma Membrane Part & $1.21 \times 10^{-19}$ & $1.69 \times 10^{-16}$ \\
\hline Molecular Function & $6.43 \times 10^{-12}$ & $6.43 \times 10^{-9}$ \\
\hline Signal Transducer Activity & & $1.20 \times 10^{-6}$ \\
\hline Molecular Transducer Activity & & \\
\hline Receptor Activity & & \\
\hline & & \\
\hline
\end{tabular}

this analysis, we found the closest genes to regulating trans ceQTLs and counted the number of overlapping genes between the two species whenever orthologous genes could be identified. For regulating trans ceQTLs, 2,381 genes were found in mouse while 5,930 were found in human. The overlap of 1,745 was significant by chi-square test $\left(\mathrm{P}<2.2 \times 10^{-16}\right)$.

We also examined the effect of trans ceQTLs regulating $\mathrm{X}$ chromosomal genes. The difference in effect sizes between autosomal and Xchromosomal loci was significant for positive $\alpha\left(\mathrm{P}=10^{-2}\right)$ but much weaker than for cis ceQTLs. There were too few observations to test negative $\alpha$ (Figure 4D) on the $\mathrm{X}$ chromosome $(\mathrm{N}=3)$. The $\mathrm{X}$ chromosome attenuation phenomenon appears to be specific for cis ceQTLs.

\section{Trans ceQTLs are functionally enriched}

Contrary to a yeast eQTL dataset [16], trans ceQTLs in our original mouse $\mathrm{RH}$ dataset were enriched for $\mathrm{GO}$ categories related to transcription. We tested the enrichment of Gene Ontology categories using DAVID for 5,929 genes closest to a trans ceQTL in our human $\mathrm{RH}$ 
data at FDR $<0.25$. The top 10 categories for the biological process ontology are displayed in Table 3 alongside enrichment results from our mouse $\mathrm{RH}$ dataset at the same threshold. A Mann-Whitney rank test on the GO category scores between these two datasets provides strong evidence of similarity $\left(\mathrm{P}=4.9 \times 10^{-7}\right)$. Complete results are shown in Additional File 8 Table S3.

Categories showing enrichment in the human $\mathrm{RH}$ data included signaling, development, binding, plasma membrane, and cytoskeleton. Remarkably, many of these same categories were enriched in the mouse dataset, showing conservation of function between the two species among trans ceQTLs, particularly ion related categories. Transcription factor related categories were enriched only in the mouse RH data at FDR $<0.25$. However, transcription factor activity was enriched in the human RH data at FDR $<0.3$.

\section{Regulatory loci in noncoding regions}

At FDR $<0.25$, a total of 1,128 out of 17,347 (6.5\%) of trans ceQTLs mapped to noncoding regions of the human genome. We considered a ceQTL as noncoding if it was $>300 \mathrm{~kb}$ away from a known gene or microRNA according to UCSC's hg18 or mm7 gene location tables. The choice of a $300 \mathrm{~kb}$ cutoff is somewhat arbitrary, but it exceeds twice the $-2 \log _{10} P$ support radius (i.e., the width of the peak two $-\log _{10} P$ units from the maximum) used in this study.

Assuming that closely linked ceQTL peaks represent individual loci regulating multiple genes, we merged noncoding ceQTLs if the peaks were $<300 \mathrm{~kb}$ from each other (Methods). At FDR $<0.25$, there were 325 noncoding ceQTLs in human and 370 in mouse. Since some noncoding ceQTLs in mouse map to more than one noncoding ceQTL in human (and vice versa), we enforced a rigorous one-to-one mapping of mouse noncoding blocks to human noncoding blocks resulting in 369 possible noncoding blocks in common between the two species. Chi-square analysis of the number of shared blocks containing ceQTLs (118) between mouse and human (205 and 199, respectively) between the two species was not significant. At a more liberal threshold of FDR $<0.3$, the overlap of noncoding blocks was significant $\left(\mathrm{P}=10^{-7}\right)$. Figure 5 shows the co-localization of mouse and human noncoding ceQTL blocks on the human genome.

We applied Gene Set Enrichment Analysis [17] to the genes regulated by the eight syntenic noncoding ceQTLs with the highest $-\log _{10} P$ values in both mouse and human datasets $\left(-\log _{10} P>4\right)$. No pair of mouse-human gene lists regulated by a common ceQTL had an overlap in their enriched GO categories. However, we found one noncoding ceQTL located on the mouse $\mathrm{X}$ chromosome at $20.6 \mathrm{Mb}$ and the syntenic region of the human $\times$ chromosome at $115.9 \mathrm{Mb}$ that affected expression of an overlapping set of gene targets regulated by 19 microRNAs $\left(\chi^{2}=8.74,1\right.$ d.f., $\left.P=3.1 \times 10^{-3}\right)$. The noncoding ceQTL itself did not harbor any microRNAs according to MiRscan (see below).

\section{microRNAs in noncoding regions}

The existence of noncoding trans ceQTLs suggested there may be unknown genomic elements in those regions. One possibility included unidentified microRNAs. We used MiRscan [18] to screen the positionally conserved noncoding ceQTLs with FDR $<0.25$. No regions resulted in significant MiRscan scores.

\section{Known noncoding elements do not explain noncoding ceQTLs}

Several recent reports using next-generation RNA-Seq and ChIP-Seq methods have found evidence of novel

Table 3 Functional enrichment of trans ceQTLs at FDR $<0.25$

\begin{tabular}{|c|c|c|c|c|c|}
\hline Human & $P$ value & FDR & Mouse & $P$ value & FDR \\
\hline \multicolumn{6}{|l|}{ Biological Process } \\
\hline System Development & $1.69 \times 10^{-25}$ & $2.70 \times 10^{-22}$ & System Development & $4.48 \times 10^{-15}$ & $6.82 \times 10^{-12}$ \\
\hline Anatomical Structure Morphogenesis & $1.21 \times 10^{-18}$ & $1.92 \times 10^{-15}$ & Organ Development & $3.42 \times 10^{-13}$ & $5.25 \times 10^{-10}$ \\
\hline Cell Differentiation & $2.96 \times 10^{-14}$ & $4.73 \times 10^{-11}$ & Anatomical Structure Morphogenesis & $6.17 \times 10^{-13}$ & $9.47 \times 10^{-10}$ \\
\hline Organ Development & $7.90 \times 10^{-13}$ & $1.26 \times 10^{-9}$ & Neuron Projection Development & $2.36 \times 10^{-11}$ & $3.63 \times 10^{-8}$ \\
\hline Ion Transport & $6.86 \times 10^{-12}$ & $1.09 \times 10^{-8}$ & Axon Guidance & $6.83 \times 10^{-11}$ & $1.05 \times 10^{-7}$ \\
\hline Cell-Cell Signaling & $1.39 \times 10^{-11}$ & $2.21 \times 10^{-8}$ & Cell Differentiation & $1.17 \times 10^{-9}$ & $1.79 \times 10^{-6}$ \\
\hline Negative Regulation Of Biological Process & $3.06 \times 10^{-11}$ & $4.89 \times 10^{-8}$ & Negative Regulation Of Cellular Process & $5.50 \times 10^{-9}$ & $8.44 \times 10^{-6}$ \\
\hline Regulation Of Multicellular Organismal Process & $4.60 \times 10^{-10}$ & $7.34 \times 10^{-7}$ & Cell Projection Morphogenesis & $5.98 \times 10^{-9}$ & $9.17 \times 10^{-6}$ \\
\hline Cell Motion & $5.61 \times 10^{-9}$ & $8.96 \times 10^{-6}$ & Negative Regulation Of Biological Process & $9.79 \times 10^{-9}$ & $1.50 \times 10^{-5}$ \\
\hline Negative Regulation Of Cellular Process & $1.27 \times 10^{-8}$ & $2.03 \times 10^{-5}$ & Cell Motion & $1.28 \times 10^{-8}$ & $1.97 \times 10^{-5}$ \\
\hline Cell Morphogenesis & $1.70 \times 10^{-8}$ & $2.72 \times 10^{-5}$ & Cell Development & $1.39 \times 10^{-8}$ & $2.14 \times 10^{-5}$ \\
\hline Transport & $2.19 \times 10^{-8}$ & $3.50 \times 10^{-5}$ & Cell Part Morphogenesis & $2.78 \times 10^{-8}$ & $4.26 \times 10^{-5}$ \\
\hline
\end{tabular}




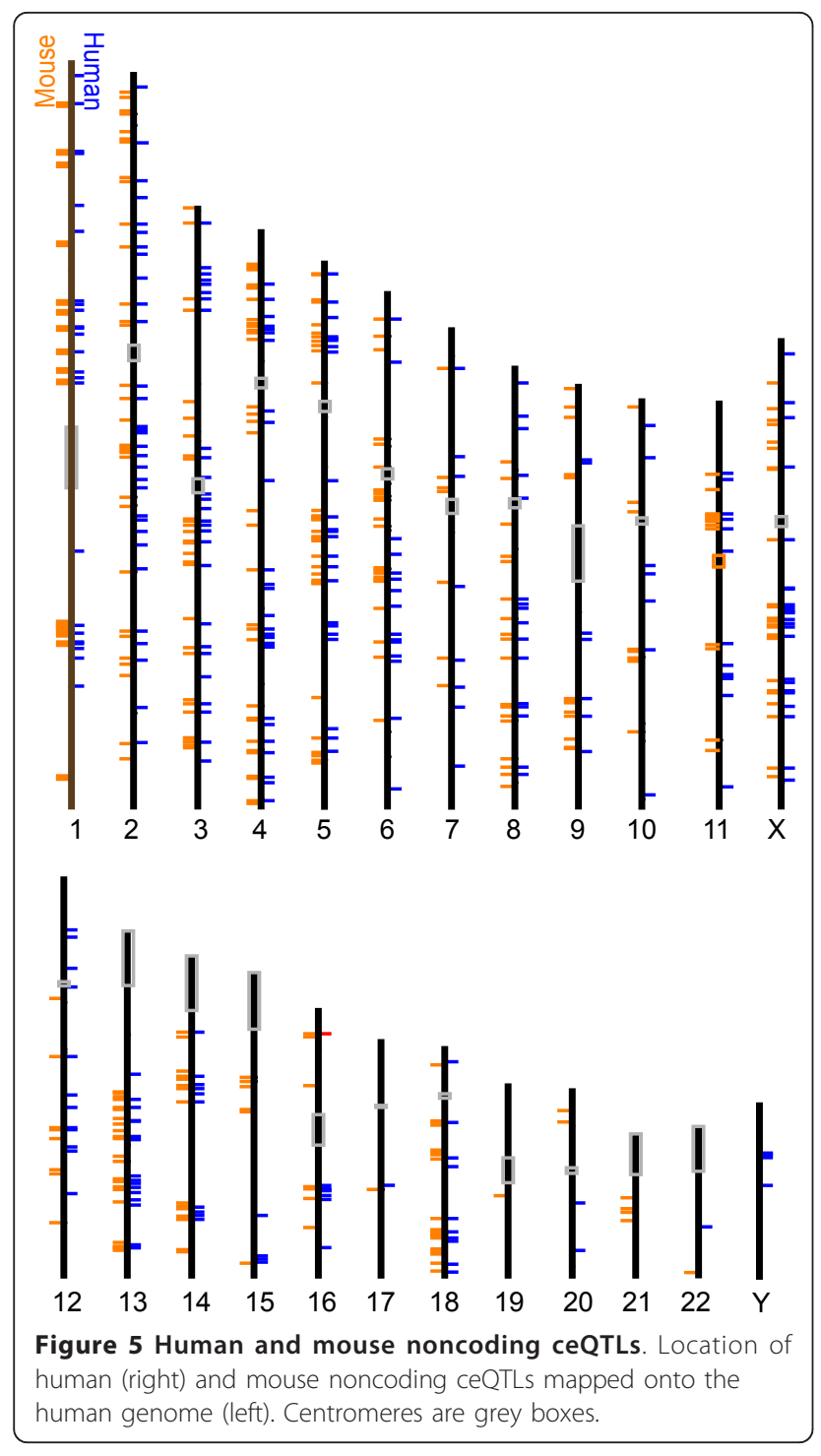

genes and functional RNAs in noncoding regions, illuminating the role of "dark DNA". We examined the positional overlap of three such datasets. The first was a deep RNA-Seq study of the mouse transcriptome which revealed evidence of novel genes [19]. In a ChIP-Seq study, a new class of large intervening noncoding RNAs (lincRNA) was identified due to the preferential association of histone $\mathrm{H} 3$ trimethylated at either lysine 4 or lysine36 with these elements [20]. Ultraconserved regions [21] are noncoding regions $>200$ bp perfectly conserved across multiple species. They possess no known function, yet appear to be under purifying selection.

The overlap between these three classes of newly discovered functional elements and our human noncoding ceQTLs at FDR $<0.25$ was sparse $(4 \%$ overlap; $96 \%$ of noncoding ceQTL blocks unexplained) (Figure 6A, B).

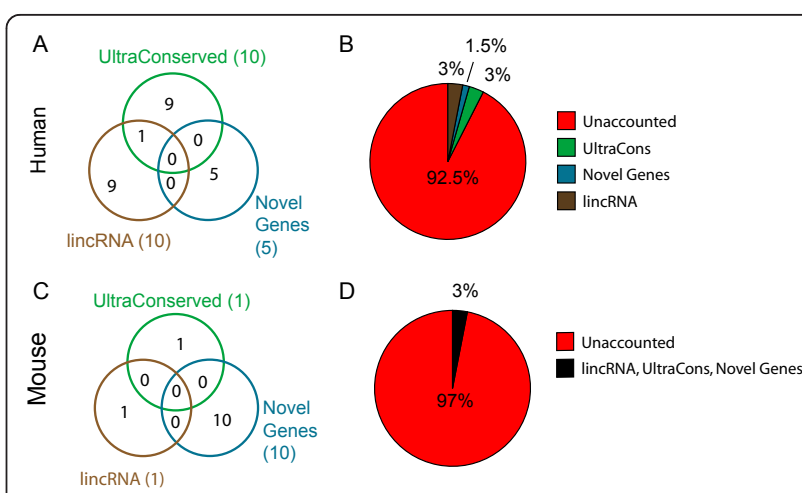

Figure 6 Trans ceQTLs in noncoding regions are not explained by recently discovered functional elements. (A) Venn diagram of the number of noncoding ceQTLs in human at FDR $<0.25$ that can be accounted for by lincRNAs, novel genes and ultra conserved regions. For example, 10 noncoding ceQTL blocks contain lincRNAs, one of which overlaps with noncoding ceQTL blocks containing ultra conserved regions. (B) The majority of the 325 noncoding ceQTL blocks in human at FDR $<0.25$ do not contain lincRNAs, novel genes or ultra conserved regions. (C) Number of noncoding ceQTL blocks in mouse at FDR $<0.25$ that can be explained by recently discovered genomic elements. (D) Of 370 noncoding ceQTLs in mouse, $3 \%$ can be accounted for by recently discovered functional elements.

Only $3.2 \%$ of the mouse RH noncoding ceQTL blocks overlap with these same elements (Figure 6C, D). Although we used a relatively liberal FDR $<0.25$ to identify the $>320$ noncoding ceQTLs, the number of true noncoding ceQTLs is still expected to be $\sim 240$. This greatly exceeds the number of lincRNAs, ultraconserved regions and novel genes, suggesting these elements cannot be the regulatory elements underlying most of our noncoding ceQTLs. The $-\log _{10} P$ values of the trans noncoding ceQTLs closest to either lincRNAs or novel genes were among the lower scoring (Additional File 9 Figure S6), implying that the majority of the noncoding ceQTLs represent novel but still undefined biological regulators.

While enhancers are known to affect gene expression at a distance, none of the non-coding ceQTLs can represent these regulatory elements. Unlike meiotic mapping, a breakpoint in $\mathrm{RH}$ mapping physically separates a regulatory element from its corresponding gene. The element is instead placed next to a randomly selected gene in each $\mathrm{RH}$ clone and will not act as a consistent trans regulatory locus.

\section{Comparison of RH data with normal tissues}

In order to evaluate our artificial human RH system against an in vivo biological data set, we compared the gene expression from the RH experiments to the human Novartis SymAtlas [22], a compendium of gene expression across multiple tissues. Using a common set of 
12,368 genes, we constructed a correlation matrix of expression for gene pairs across the $\mathrm{RH}$ panel and a similar matrix across the 79 tissues of the SymAtlas. We then subtracted the two matrices and computed the Frobenius norm (Methods) to quantify the distance between the two data sets. To generate a null distribution, the gene expression values from the $\mathrm{RH}$ data were permuted, a new correlation matrix was computed and subtracted from the SymAtlas correlation matrix and the Frobenius norm recomputed. Of 10,000 permutations, none showed a score smaller than the observed score $\left(P<10^{-4}\right)$ (Additional file 10 Figure S7). This result suggests that pair-wise gene expression changes obtained from copy number variation in the $\mathrm{RH}$ panel are similar to those obtained from regulated gene expression in multiple tissues of a mammalian organism.

\section{Discussion}

The relationship between copy number and gene expression has only begun to be explored as most studies are focused on identifying regions of copy number variation (CNV) [23-25]. The first studies to extensively explore CNV effects on expression in mice highlighted the potential for widespread impact of CNVs on shaping the transcriptome of various tissues $[6,26]$. Recent studies of CNV effects on gene expression in human and mouse rely upon naturally occurring variation (deletions, duplications, triplications, etc) and have been limited to cis effects $[27,28]$. Radiation hybrid panels allow a genome-wide survey of gene expression changes due to copy number increases and are not limited to regions of previously identified CNVs.

Several lines of evidence support the broader applicability of RH panels in understanding gene expression networks. Though highly multiplexed, $\mathrm{RH}$ panels are not unlike other systems such as transgenic organisms or transfected cell lines which have given useful biologically insights. Phenotypic mapping experiments using radiation hybrids have successfully located human and murine viral entry proteins [29-32] by exploiting the ability of $\mathrm{RH}$ clones to correctly express exogenous genes and synthesize and post-translationally modify the resulting proteins. Recent sequencing efforts of the hamster genome showed that coding sequences are $88 \%$ conserved with human [33].

The gene-gene correlation between human $\mathrm{RH}$ and SymAtlas datasets also implies no substantial difference in gene expression between our human $\mathrm{RH}$ panels and in vivo gene expression for the 12,000 genes we tested. One caveat is their different sources of genetic variation so this result should be considered in context with other available evidence. Unlike genetic coexpression studies, the high resolution of the $\mathrm{RH}$ approach allowed construction of directed genetic networks from the mouse
RH data. These directed networks showed significant overlap with other networks including protein-protein interaction and coexpression networks [34]. Adding the human $\mathrm{RH}$ data will improve the resolution and power of the directed $\mathrm{RH}$ genetic networks giving additional insights into the hierarchical circuitry of gene regulation.

Using a human-hamster $\mathrm{RH}$ panel, a mouse-hamster $\mathrm{RH}$ panel, an aneuploid mouse dataset and publicly available TCGA data, we present strong evidence that many genes possess the ability to decrease their gene expression in response to increased copy number (i.e., possess negative cis $\alpha$ ). In the mouse and human $\mathrm{RH}$ datasets, $30 \%$ of genes show this ability compared to $6 \%$ of surveyed TCGA genes. Some of this is likely due to the difference in coverage: the entire human/mouse genome was represented in the $\mathrm{RH}$ panels while only $38 \%$ of the genome was covered in TCGA data. A small number of negative cis alphas have been reported in human $[5,8,27]$ and mouse [26,28], but the RH approach is the first to interrogate the entire mammalian genome.

Additional factors may underlie some of these negative cis ceQTLs, but are unable to account for the totality of negative cis ceQTLs. Antisense transcription plays no significant role and the inclusion of partial length genes in each $\mathrm{RH}$ clone could maximally account for only a minority $(<21 \%)$ of negative cis ceQTLs.

Across the RH and TCGA data, the most enriched gene ontology categories for genes that decrease expression in response to increased copy number involved signaling, receptor activity and membrane functions. This finding is new and suggests that signaling pathways are tightly regulated and may possess autoregulatory feedback to compensate for increased copy number. Signaling genes were recently found to be enriched among human CNVs [24] and under positive selective pressure [35], possibly because negative cis $\alpha$ values confer a regulatory robustness in the face of sequence changes. Study of individual genes should reveal details of the responsible mechanisms.

We found 42 common genes with negative cis $\alpha$ between the two RH and TCGA data sets (Additional file 2 Table S2). Surprisingly, the relatively modest overlap in the number of genes still yields a high degree of similarity in GO categories across the three data sets suggesting conserved pathways are affected.

We observed that cis ceQTLs on the human X chromosome showed substantially lower effect sizes than autosomes - a discovery we first noted in the mouse $\mathrm{RH}$ panel. The attenuation of the relationship between dosage and expression is independent of Xist mediated $\mathrm{X}$ chromosome inactivation and may represent a form of previously unseen dosage compensation in mammals. In placental mammals, $\mathrm{X}$ chromosome inactivation occurs through the expression of Xist, a noncoding 
RNA on the future inactive $\mathrm{X}$ chromosome (Xi) [36]. Transcribed sequences from the Xist locus coat the $\mathrm{Xi}$ elect by binding nongenic regions of the $\mathrm{X}$ chromosome $[37,38]$. The predicted secondary RNA structure of Xist possesses two stem loops and may serve as a scaffold for silencing factors [39]. Chromatin modification [40], scaffold proteins [41], and polycomb proteins [42] have all been implicated in the initiation and maintainance of $\mathrm{X}$ chromosome inactivation although the picture is far from clear. In contrast to mammals, Drosophila [43] and C. elegans [44] both use transcriptional control for $\mathrm{X}$ chromosome dosage compensation. The autoregulatory control of $\mathrm{X}$ chromosome expression found in the human and mouse RH panels may thus represent an evolutionary remnant of these invertebrate dosage compensation mechanisms which has since been supplemented by $\mathrm{X}$ chromosome inactivation. The same attenuation pattern was found in male TCGA data on the $\mathrm{X}$ chromsosome. While cancer resembles $\mathrm{RH}$ clones in some respects, cancer cells differ in several important aspects such as mutation, selection, heterogeneity of fragment length and differences in genome coverage.

Among trans loci, we found evidence of conserved regulating genes between the human and mouse $\mathrm{RH}$ panels. Trans ceQTLs were particularly associated with genes involved in binding, signaling and ion-channel activity suggesting that these genes tend to represent network hubs and that copy number changes in these genes can contribute to non-lethal variation. We found enrichment of transcription factor activity in mouse but not human RH data at FDR $<0.25$. However, at FDR $<$ 0.3 , transcription factor activity was enriched in human $\mathrm{RH}$ as well. Trans regulatory hotspots have been observed in eQTL studies involving yeast [16], mouse [45] and human [46] and are commonly interpreted as evidence for master regulators. However, unanticipated factors in the data may contribute to false positives. For instance, a high degree of relatedness between mouse strains has produced signatures of regulatory hotspots [47] and association with groups of highly correlated genes has produced unlikely regulatory hotspots $[48,49]$. Integrating additional information such as transcription factor binding sites, protein-protein interaction data and functional analysis is helpful in identifying likely candidates when unanticipated heterogeneity may exist $[48,50]$.

We found noncoding ceQTLs in both human and mouse. Debate continues about the importance of the substantial portion of the genome that does not code for genes. While it is clear that much of the genome is actively transcribed, the role of these regions is unclear. We examined new datasets containing genes and functional genomic elements in noncoding regions, yet the vast majority of our noncoding ceQTLs cannot be explained by these recent discoveries. We also found no significant overlap of the location of noncoding ceQTL blocks in both species at FDR $<0.25$. At a slightly less stringent $\mathrm{FDR}<0.3$, there is significant overlap in the locations of noncoding ceQTL blocks in both species but the regulated genes differ. This may reflect evolutionary divergence. Indeed, microRNAs, many of which are conserved across species, have also been found to show species-specific regulation [51].

Our own search for novel microRNAs in noncoding ceQTLs yielded no candidates, though it is likely that improved screening techniques and computational algorithms may aid their discovery. Also, there were very small numbers of other unconventional RNAs such as linc RNAs in the noncoding ceQTLs. Thus, unanticipated forms of gene regulation seem likely. While the RH approach does not reveal possible mechanisms of action, the noncoding ceQTL data could act as a guide for discovery of these novel elements by allowing transfection of overlapping genomic DNA fragments traversing the ceQTL combined with transcript profiling as a bioassay.

Radiation hybrid panels exist for a number of other organisms including sheep [52], pig [53], cow [54,55], rat [56] and dog [57]. The potential exists for probing species-specific copy number effects on gene expression. Amalgamating these data sets can also be used to improve mapping resolution and examine common networks of gene regulation and regulatory regions.

\section{Conclusions}

Radiation hybrid panels are a valuable tool for probing the relationship between copy number and gene expression in the mammalian genome in a largely unbiased manner. In both human and mouse radiation hybrid panels, we have mapped to high resolution cis and trans loci capable of affecting gene expression due to copy number change and found a number of consistent results. Approximately $30 \%$ of genes show an inverse correlation between increased copy number and gene expression and genes on the $\mathrm{X}$ chromosome show an attenuated response to copy number increase as compared to autosomes, suggesting a potentially novel form of dosage compensation. Copy number perturbations of noncoding regions were shown to affect gene expression as well and the lack of known control elements in these regions may imply novel regulatory loci.

\section{Methods \\ Cells}

RH clones were thawed and cultivated in alpha-MEM with $10 \%$ FBS, $1 \mathrm{X}$ ampicilin and $1 \mathrm{X}$ HAT. Cells were trypsinized and DNA and RNA harvested as described in our previous study [8]. 


\section{Microarray analysis}

RNA from each of the 80 available radiation hybrid clones and A23 recipient hamster cell line was hybridized in duplicate (technical replicates) to single channel Illumina HumanRef-8 v1.0 BeadChips by the UCLA Southern California Genotyping Consortium according to manufacturer's protocols. The raw data was extracted using Illumina BeadStudio v1.5.1 and median normalized using Genespring GX (Agilent). Duplicate array measurements were log averaged prior to the construction of $\mathrm{RH}$ to A23 ratios for 20,996 genes.

The average correlation between replicates was $r=0.92$ $\left(P<2.2 \times 10^{-16}\right)$. Hierarchical clustering always grouped duplicate arrays together (Additional File 2 Figure S2).

\section{Comparative Genomic Hybridization}

DNA from each radiation hybrid clone was extracted and hybridized to Agilent 244 K human comparative genomic hybridization $(\mathrm{aCGH})$ arrays which contain 60 mer oligonucleotide probes. Hamster A23 DNA, serving as the control, was the other channel. Arrays were labeled and scanned according to manufacturer's instructions.

\section{Normalization of aCGH data}

Preprocessing and normalization of the raw aCGH data was performed as described previously [8]. Briefly, raw aCGH intensity data (RH/A23) for 235,829 markers was $\log _{10}$ transformed and then averaged over a sliding window of ten adjacent markers for each cell line. The bimodal distribution of $\log _{10}$ intensity across all cell lines (Additional File 11 Figure S8A-B) showed markers with no copy number increase and those with an increase of one or more copies. Most copy number changes were an increase of one with the probability of retaining two copies $\sim 1 \%$.

Since the recipient hamster cells are male, the copy number increase for the autosomes was three compared to two and two compared to one for the sex chromosomes. We therefore normalized the $\log _{10}$ transformed aCGH data by centering the first mode at zero $\left(\log _{10}(2 /\right.$ 2)) and then scaling the data so that the second mode was centered at $\log _{10}(3 / 2)$ for the autosomes and $\log _{10}$ $(2 / 1)$ for the sex chromosomes.

To quantify marker loss/gain compared to the legacy PCR data as a result of passaging of G3 radiation hybrid clones, we averaged the $\log _{10}(\mathrm{RH} / \mathrm{A} 23)$ aCGH ratio for the ten markers closest to a STS marker. If this value exceeded the $95^{\text {th }}$ percentile of the first mode of the omnibus distribution, we classified this region as retained. Of the 80 available G3 clones, one clone did not match any PCR genotypes and was excluded from all subsequent analyses.

\section{Linear model}

We used a linear model to characterize the change in gene expression due to increased copy number [8]. For each gene, we modeled the data as $y=\mu+\alpha x$ where $y$ is the normalized $\log _{10}(\mathrm{RH} / \mathrm{A} 23)$ expression, $x$ is the $\log _{10}(\mathrm{RH} / \mathrm{A} 23)$ aCGH data, $\mu$ is the baseline gene expression and $\alpha$ is the ordinary least squares estimate reflecting the effect size. This model was compared with a reduced model $y=\mu$ exactly like an F-test, except that permutation was used to generate a null distribution of residuals and assign P values. We tested 20,996 genes and 235,829 markers, to calculate all $4,951,465,684$ possible combinations.

The permutation employed random re-assortment of the gene expression data and recalculation of the F-statistic five times for each combination $(5 \times 20,996 \times$ $235,829)$. The correlation structure of the markers was retained between each permutation. The pooled F-statistics served as the empirical null and $P$ values were calculated as the frequency of null values greater than the observed F statistic.

The Benjamini-Hochberg method was used to control false discovery rates (FDRs). Since cis ceQTLs (marker < $5 \mathrm{Mb}$ from a gene) test a different set of hypotheses than trans ceQTLs (marker $>5 \mathrm{Mb}$ from a gene), we applied FDR separately to cis and trans ceQTLs.

We also utilized the R/Bioconductor package DNAcopy to bin $\mathrm{CGH}$ data into 0 or 1 extra copies of the donor locus in order to assess species specific hybridization artifacts. Copy number, instead of CGH intensity, was then used in the linear model. A comparison of $\alpha$ 's obtained by our original procedure and using the binned CGH data showed excellent concordance $(r=0.95, \mathrm{P}<$ $\left.2.2 \times 10^{-16}\right)$ (Additional File 12 Figure S9) .

\section{TCGA data analysis}

We downloaded matched aCGH and expression data $(\mathrm{N}$ = 237) from The Cancer Genome Atlas (TCGA, http:// tcga.cancer.gov) glioblastoma multiforme data portal. These data were normalized by the TCGA consortium. TCGA aCGH data consists of a tumor sample hybridized to one channel and a male reference sample hybridized to the other channel. Because only $38.8 \%$ of autosomes and $68 \%$ of the $\mathrm{X}$ chromosome are affected by copy number perturbation in this data set, we discarded aCGH markers that did not show a change in copy number (e.g., $>\log _{2}(3 / 2)$ or $<\log _{2}(1 / 2)$ for autosomes) in at least one sample. For each CGH marker within a $5 \mathrm{Mb}$ radius, we performed linear regression to estimate the effect of increased copy number on gene expression. An FDR correction was applied to the data such that all TCGA results have a FDR $<0.05(P<$ 0.04). 


\section{Comparison of cis ceQTL overlap between data sets}

To compare the overlap of ceQTLs with positive and negative cis $\alpha$ between human and mouse RH data, we performed a chi-square test of a $2 \times 2$ contingency table in R. Orthologous genes between the two data sets were first identified and then we counted the number of cis ceQTLs with positive and negative $\alpha$ in both data sets as well as those that those that were positive in one data set and negative in the other. Comparison of the positive-positive and negative-negative cells of the $2 \times 2$ table with the expected counts indicated enrichment. The same procedure was used for comparing human RH with mouse trisomic and TCGA data.

\section{Evaluation of hamster transcripts on human microarrays} Exploiting the high conservation of coding sequences in mammals, we used a human microarray platform to interrogate the expression of the donor human and recipient hamster genes in the G3 RH panel. Illumina BeadChip probes consist of relatively long (50 mer) oligonucleotides potentially allowing evaluation of transcripts from both species.

We tested whether the expression arrays could detect hamster and human transcripts with comparable efficiency. RNA extracted from hamster and human liver, kidney and heart and compared the relative expression signals as a ratio (Additional File 1 Figure S1A). The bulk of the ratios were centered around zero for $\log _{10}$ (human/hamster) expression, indicating equivalent performance in measuring hamster and human expression for most genes. As expected, some probes showed a preference for human transcripts.

While species differences in sequence hybridization may influence detection of cis ceQTLs, detection of trans ceQTLs is not directly affected by such variation. We therefore compared the distributions of $\log _{10}$ human/hamster tissue gene expression for cis-and trans-regulated genes, with the trans ceQTL distribution acting as a control. The difference in expression ratios between the two groups was not large (Additional File 1 Figure S1B-D). As expected, there was some preference for human genes (18.8\%). Based on this evidence, most of our cis ceQTLs $(>80 \%)$ are not due to differences in hybridization on the microarray.

To evaluate array CGH use for hamster, we co-hybridized genomic DNA from hamster and human to the array and evaluated the signal intensities for each channel separately. Correlation between the two species on the aCGH array was 0.57 and the means of the human and hamster signals are quite close (6.7 and 7.0 respectively). For human, the signal intensity has a larger standard deviation than hamster $(0.88$ versus 0.37 respectively) (Additional File 1 Figure S1E-F). Preferential binding of human DNA would lead to a conservative bias as it minimizes signal from the hamster genome which is expected to be unperturbed.

\section{Trans Hotspot FDR}

We calculated the probability that a marker would regulate more than $n$ genes using a Poisson distribution with mean equal to the average number genes regulated by trans ceQTLs across all markers. These $\mathrm{p}$-values were then subjected to Benjamini-Hochberg correction to obtain an FDR. Similarly, the probability that a gene is regulated by more than $m$ ceQTLs was modeled as Poisson with mean equal to the average number of markers regulating each gene. These $\mathrm{p}$-values were FDR corrected as above.

\section{Noncoding regions}

We defined a trans ceQTL as noncoding if $>300 \mathrm{~kb}$ away from a known gene or microRNA using the UCSC human hg18 (NCBI 36.1) or mouse mm7 (NCBI 35.1) gene and microRNA tables. The UCSC gene set is larger $(\sim 50,000$ entries) and less conservative than RefSeq ( 20,000 entries), including genes with alternative start sites, alternatively spliced exons and putative but unknown genes. CeQTL peaks in noncoding regions are likely due to the same genes if nearby and were merged together if within $300 \mathrm{~kb}$ in both human and mouse datasets.

\section{Conversion of mouse locations to human locations}

UCSC's LiftOver utility allows the conversion of genome coordinates from one species to another, using whole genome alignments (nets and chains) generated by their BLASTZ analysis [58,59]. The 232,626 mouse markers were subjected to the recommended minMatch parameter of 0.10 for interspecies conversion using $\mathrm{mm} 7$ to hg18 liftover chain files. Approximately 160,000 markers were converted at this level.

\section{microRNA discovery}

We essentially followed the published methodology for using MiRscan [18]. First, we employed the RNAfold program from the Vienna RNA software package $[60,61]$ and scanned $100 \mathrm{nt}$ windows in our mouse noncoding regions to find regions of stable hairpin formation. As a cutoff, we used a minimum free energy value of -25 $\mathrm{kcal} / \mathrm{mol}$. All candidate regions were BLASTed against human noncoding regions to find the best matching region, which was then fed to RNAfold to determine their minimum hairpin free energy. Regions meeting the same minimum free energy value of $-25 \mathrm{kcal} / \mathrm{mol}$ were passed to MiRscan, which uses multiple conservation criteria to score a region for possible microRNA content. Of the $\sim 325$ noncoding regions tested, none were significant. 


\section{Comparison with SymAtlas}

The Novartis SymAtlas [22] contains expression values for $\sim 22,000$ probes across 79 different human tissues. A common set of 12,368 genes were identified between the SymAtlas and the human RH data. Two separate gene-gene correlation matrices were constructed based on the expression data, one each for RH and SymAtlas. We then subtracted the two correlation matrices from each other to obtain the matrix $A$ and computed the Frobenius norm defined as

$$
\|A\|_{F}=\sqrt{\sum_{i} \sum_{j}\left|a_{i j}\right|^{2}}
$$

which serves as a distance measurement between the two correlation matrices. A low score represents high similarity. To generate a null data set, we permuted the assignment of expression values in the human $\mathrm{RH}$ data, created a new correlation matrix, subtracted the matrix from the SymAtlas matrix and recomputed the Frobenius norm 10,000 times. The $P$ value is determined by the number of times a permuted score is less than our observed score. The gene expression correlation structures were preserved in the permuted matrices.

\section{Data Availability}

Expression microarray and array comparative genomic hybridization $(\mathrm{aCGH})$ data were submitted to the Gene Expression Omnibus under accession number GSE19003.

\section{Software}

To automate, parallelize and optimize the computational analysis, custom Perl and C programs and modules were written to handle data and manage applications. Standalone BLAST was used to create custom sequence databases and Bioperl packages [62] used to automate BLAST queries and manipulate sequence data. BLAT [63] was used to obtain genome coordinates for microarray probes.

\section{Additional material}

Additional file 1: Figure S1. Evaluation of human microarrays. (A) $\log _{10}$ (human/hamster) expression ratios averaged across kidney, heart and liver. (B) $\log _{10}$ (human/hamster) expression ratios for genes regulated by both cis (pink) and trans (blue) ceQTLs. The overlap between the two distributions is purple. (C) $\log _{10}$ (human/hamster) expression ratios for genes regulated by cis ceQTLs. (D) $\log _{10}$ (human/hamster) expression ratios for genes regulated by trans ceQTLs. (E) aCGH signal distribution for hamster and (F) human.

Additional file 2: Figure S2. Expression arrays showed good replicability. Hierarchical clustering of expression arrays always placed duplicates next to each other. Duplicates referred to as ' $a$ ' and ' $b$ '.

Additional file 3: Figure S3. Retention frequency based on aCGH data. (A) aCGH intensity data for human RH clone 12 along chromosome 2 matches historical PCR data well (red lines) but does show some loss. (B) Retention frequency of human donor genome across all $79 \mathrm{RH}$ clones. Solid line is loess smoothed with parameter 0.02. (C) Retention frequency of chromosome 6 is relatively uniform except for the centromere (grey) which shows preferential retention. (D) The Tk1 gene (red arrow) is retained at $100 \%$ as expected for the selectable marker. (E) The difference in retention frequency between centromeric (grey bars) and noncentromeric (red bars) region for all chromosomes is statistically significant (Welch's $t>8.1$, d.f. $>477, P<10^{-15}$ ). (F) The $X$ chromosome has $\sim 50 \%$ retention frequency of the autosomes because the donor cell line was male. The $Y$ chromosome has an apparently higher retention frequency than the $X$, probably because the $Y$ has a proportionally higher percentage of centromeric sequence (cf. Figure S4E). Error bars $s$. e.m.

Additional file 4: Figure S4. Mapping resolution and effect sizes. (A) The median distance between a human gene and its cis ceQTL at FDR < 0.4 is $531 \mathrm{~kb}$. (B) Distribution of human cis ceQTL $\alpha$ values. Positive $\alpha$ indicates induction of gene expression due to copy number increase, while negative $\alpha$ indicates repression.

Additional file 5: Table S1. GO Enrichment for positive cis $\alpha$ at FDR $<$ 0.25 .

Additional file 6: Figure S5. Comparison of genes with positive and negative cis $\alpha$. (A) Histogram of expression values for genes with positive cis $\alpha$ (pink) and negative cis $\alpha$ (blue) with means 12.04 and 11.99 respectively. The overlap is in purple. (B) Occurrence of full length genes across all $79 \mathrm{RH}$ clones. Each gene is found in its entirety on average 6 times. 3,422 genes are never found in their entirely across all clones. (C) Scatterplot of cis $\alpha$ 's derived from the peak marker and its neighbor ( $r=$ $\left.0.99, \mathrm{P}<2.2 \times 10^{-16}\right)$. (D) Cis $\alpha^{\prime}$ s of the peak marker and the $5^{\text {th }}$ closest marker ( $75 \mathrm{~kb}$ away). Correlation is $0.96\left(P<2.2 \times 10^{-16}\right)$.

Additional file 7: Table S2. Common genes with negative cis $\alpha$ in mouse RH, human RH and TCGA.

Additional file 8: Table S3. Functional enrichment of trans ceQTLs at FDR $<0.25$.

Additional file 9: Figure S6. Distribution of $-\log _{10} P$ values for ceQTLs in human noncoding regions. Noncoding ceQTLs closest to known lincRNAs and recently discovered unconventional genes are indicated by red arrows and tend to be among the $\operatorname{lower}_{-}-\log _{10} P$ values.

Additional file 10: Figure S7. Comparison between human $\mathrm{RH}$ and SymAtlas. Distribution of Frobenius norm values for distance between human RH and SymAtlas data using permuted expression values. Observed human RH-SymAtlas distance shown in red. Units are arbitrary.

Additional file 11: Figure S8. $\log _{10}(\mathrm{RH} / \mathrm{A} 23) \mathrm{aCGH}$ intensity data is bimodal. (A) Histogram of aCGH intensity for all RH clones. The large mode represents equivalent copy number between $\mathrm{RH}$ clones and hamster A23 control genomes, while the smaller mode to the right indicates markers with an extra copy in the $\mathrm{RH}$ clones. (B) Close up view of the second mode.

Additional file 12: Figure S9. Comparison of $\alpha$ from binned and continuous CGH data. CGH data was either binned into 0 or 1 extra copies or used as continuous values and used to calculate $\alpha$. The correlation is $0.95, \mathrm{P}<2.2 \times 10^{-16}$.

\section{Acknowledgements and Funding}

We thank Dusty Miller of the Fred Hutchinson Cancer Research Center for providing the G3 RH panel. This work was supported by National Human Genome Research Institute T32-HG0002536 and the Stein Oppenheimer Endowment Award, UCLA.

\section{Author details}

'Department of Molecular and Medical Pharmacology, David Geffen School of Medicine, University of California, Los Angeles, CA 90095, USA.

${ }^{2}$ Department of Electrical Engineering, Signal and Image Processing Institute, School of Engineering, University of Southern California, Los Angeles, CA 90089, USA. ${ }^{3}$ Department of Biostatistics, School of Public Health, University 
of California, Los Angeles, CA 90095, USA. ${ }^{4}$ Department of Human Genetics, David Geffen School of Medicine, University of California, Los Angeles, CA 90095, USA. ${ }^{5}$ GE Global Research Center, One Research Circle KW-C1308, Niskayuna, NY 12309, USA.

\section{Authors' contributions}

R.T.W., A.H.K., C.C.P. carried out experiments. R.T.W., S.A., K.L. analyzed data. R. T.W., D.J.S. wrote the paper. D.J.S. designed research. All authors have read and approved the final manuscript.

\section{Received: 8 August 2011 Accepted: 16 November 2011}

Published: 16 November 2011

\section{References}

1. Goss $\mathrm{SJ}$, Harris $\mathrm{H}$ : New method for mapping genes in human chromosomes. Nature 1975, 255(5511):680-684.

2. Cox DR, Burmeister M, Price ER, Kim S, Myers RM: Radiation hybrid mapping: a somatic cell genetic method for constructing highresolution maps of mammalian chromosomes. Science 1990, 250(4978):245-250.

3. Sebat J, Lakshmi B, Troge J, Alexander J, Young J, Lundin P, Maner S, Massa H, Walker M, Chi M, et al: Large-Scale Copy Number Polymorphism in the Human Genome. Science 2004, 305(5683):525-528.

4. Redon R, Ishikawa S, Fitch KR, Feuk L, Perry GH, Andrews TD, Fiegler H, Shapero MH, Carson AR, Chen W, et al: Global variation in copy number in the human genome. Nature 2006, 444(7118):444-454.

5. Lee JA, Madrid RE, Sperle K, Ritterson CM, Hobson GM, Garbern J, Lupski JR, Inoue K: Spastic paraplegia type 2 associated with axonal neuropathy and apparent PLP1 position effect. Ann Neurol 2006, 59(2):398-403.

6. Cahan P, Li Y, Izumi M, Graubert TA: The impact of copy number variation on local gene expression in mouse hematopoietic stem and progenitor cells. Nat Genet 2009, 41(4):430-437.

7. Deeb SS: The molecular basis of variation in human color vision. Clin Genet 2005, 67(5):369-377.

8. Park CC, Ahn S, Bloom JS, Lin A, Wang RT, Wu T, Sekar A, Khan AH, Farr CJ, Lusis $A J$, et al: Fine mapping of regulatory loci for mammalian gene expression using radiation hybrids. Nat Genet 2008, 40:(4):421-429.

9. Stewart EA, McKusick KB, Aggarwal A, Bajorek E, Brady S, Chu A, Fang N, Hadley D, Harris M, Hussain $S$, et al: An STS-based radiation hybrid map of the human genome. Genome Res 1997, 7(5):422-433.

10. Figueroa J, Pendon C, Valdivia M: Molecular cloning and sequence analysis of hamster CENP-A CDNA. BMC Genomics 2002, 3(1):11.

11. Benjamini $Y$, Hochberg $Y$ : Controlling the false discovery rate - a practical and powerful approach to multiple testing. J Royal Stat Soc, Series B 1995, 57(1):289-300.

12. Benjamini Y, Yekutieli D: The control of the false discovery rate in multiple testing under dependency. Ann Stat 2001, 29:1165-1188.

13. Brem RB, Yvert G, Clinton R, Kruglyak L: Genetic dissection of transcriptional regulation in budding yeast. Science 2002, 296(5568):752-755.

14. Dennis G, Sherman B, Hosack D, Yang J, Gao W, Lane H, Lempicki R: DAVID: Database for Annotation, Visualization, and Integrated Discovery. Genome Biology 2003, 4(9):R60.

15. Williams BR, Prabhu VR, Hunter KE, Glazier CM, Whittaker CA, Housman DE, Amon A: Aneuploidy Affects Proliferation and Spontaneous Immortalization in Mammalian Cells. Science (New York, NY) 2008, 322(5902):703-709

16. Yvert G, Brem RB, Whittle J, Akey JM, Foss E, Smith EN, Mackelprang R, Kruglyak L: Trans-acting regulatory variation in Saccharomyces cerevisiae and the role of transcription factors. Nat Genet 2003, 35(1):57-64.

17. Subramanian A, Tamayo P, Mootha VK, Mukherjee S, Ebert BL, Gillette MA, Paulovich A, Pomeroy SL, Golub TR, Lander ES, et al: Gene set enrichment analysis: A knowledge-based approach for interpreting genome-wide expression profiles. Proceedings of the National Academy of Sciences of the United States of America 2005, 102(43):15545-15550.

18. Lim LP, Lau NC, Weinstein EG, Abdelhakim A, Yekta S, Rhoades MW, Burge CB, Bartel DP: The microRNAs of Caenorhabditis elegans. Genes and Development 2003, 17(8):991-1008.

19. Mortazavi A, Williams BA, McCue K, Schaeffer L, Wold B: Mapping and quantifying mammalian transcriptomes by RNA-Seq. Nat Methods 2008 5(7):621-628.
20. Guttman M, Amit I, Garber M, French C, Lin M, Feldser D, Huarte M, Zuk O, Carey B, Cassady J, et al: Chromatin signature reveals over a thousand highly conserved large non-coding RNAs in mammals. Nature 2009, 458(7235):223-227.

21. Bejerano G, Pheasant M, Makunin I, Stephen S, Kent WJ, Mattick JS, Haussler D: Ultraconserved Elements in the Human Genome. Science 2004, 304(5675):1321-1325.

22. Su Al, Wiltshire T, Batalov S, Lapp H, Ching KA, Block D, Zhang J, Soden R, Hayakawa $M$, Kreiman $G$, et al: A gene atlas of the mouse and human protein-encoding transcriptomes. Proc Natl Acad Sci USA 2004, 101(16):6062-6067.

23. Sudmant PH, Kitzman JO, Antonacci F, Alkan C, Malig M, Tsalenko A, Sampas N, Bruhn L, Shendure J, Project G, et al: Diversity of Human Copy Number Variation and Multicopy Genes. Science 2010, 330(6004):641-646.

24. Conrad DF, Pinto D, Redon R, Feuk L, Gokcumen O, Zhang Y, Aerts J, Andrews TD, Barnes C, Campbell P, et al: Origins and functional impact of copy number variation in the human genome. Nature 2009, 464(7289):704-712

25. Kidd JM, Cooper GM, Donahue WF, Hayden HS, Sampas N, Graves T, Hansen N, Teague B, Alkan C, Antonacci F, et al: Mapping and sequencing of structural variation from eight human genomes. Nature 2008, 453(7191):56-64.

26. Henrichsen $C N$, Vinckenbosch $N$, Zollner S, Chaignat E, Pradervand S, Schutz F, Ruedi M, Kaessmann H, Reymond A: Segmental copy number variation shapes tissue transcriptomes. Nat Genet 2009, 41(4):424-429.

27. Stranger $B E$, Forrest MS, Dunning M, Ingle CE, Beazley $C$, Thorne $N$, Redon R, Bird CP, de Grassi A, Lee C, et al: Relative impact of nucleotide and copy number variation on gene expression phenotypes. Science 2007, 315:848-853

28. Orozco LD, Cokus SJ, Ghazalpour A, Ingram-Drake L, Wang S, van Nas A, Che N, Araujo JA, Pellegrini M, Lusis AJ: Copy number variation influences gene expression and metabolic traits in mice. Human Molecular Genetics 2009, 18(21):4118-4129.

29. Rasko JE, Battini JL, Gottschalk RJ, Mazo I, Miller AD: The RD114/simian type $D$ retrovirus receptor is a neutral amino acid transporter. Proc Natl Acad Sci USA 1999, 96(5):2129-2134.

30. Rai SK, Duh FM, Vigdorovich V, Danilkovitch-Miagkova A, Lerman MI, Miller AD: Candidate tumor suppressor HYAL2 is a glycosylphosphatidylinositol (GPI)-anchored cell-surface receptor for jaagsiekte sheep retrovirus, the envelope protein of which mediates oncogenic transformation. Proc Natl Acad Sci USA 2001, 98(8):4443-4448.

31. Miller AD: Identification of Hyal2 as the cell-surface receptor for jaagsiekte sheep retrovirus and ovine nasal adenocarcinoma virus. Curr Top Microbiol Immunol 2003, 275:179-199.

32. Miller $A D$, Bergholz $U$, Ziegler $M$, Stocking $C$ : Identification of the myelin protein plasmolipin as the cell entry receptor for Mus caroli endogenous retrovirus. J Virol 2008, 82(14):6862-6868.

33. Kantardjieff A, Nissom PM, Chuah SH, Yusufi F, Jacob NM, Mulukutla BC, Yap M, Hu W-S: Developing genomic platforms for Chinese hamster ovary cells. Biotechnology Advances 2009, 27(6):1028-1035.

34. Ahn S, Wang RT, Park CC, Lin A, Leahy RM, Lange K, Smith DJ: Directed Mammalian Gene Regulatory Networks Using Expression and Comparative Genomic Hybridization Microarray Data from Radiation Hybrids. PLoS Comput Biol 2009, 5(6):e1000407.

35. Kim PM, Korbel JO, Gerstein MB: Positive selection at the protein network periphery: Evaluation in terms of structural constraints and cellular context. Proceedings of the National Academy of Sciences 2007, 104(51):20274-20279.

36. Chow JC, Yen Z, Ziesche SM, Brown CJ: Silencing of the mammalian $x$ chromosome. Annu Rev Genomics Hum Genet 2005, 6:69-92.

37. Chaumeil J, Le Baccon P, Wutz A, Heard E: A novel role for Xist RNA in the formation of a repressive nuclear compartment into which genes are recruited when silenced. Genes Dev 2006, 20(16):2223-2237.

38. Clemson CM, Hall LL, Byron M, McNeil J, Lawrence JB: The $\times$ chromosome is organized into a gene-rich outer rim and an internal core containing silenced nongenic sequences. Proc Natl Acad Sci USA 2006, 103(20):7688-7693.

39. Wutz A, Rasmussen TP, Jaenisch R: Chromosomal silencing and localization are mediated by different domains of Xist RNA. Nat Genet 2002, 30(2):167-174 
40. Lucchesi JC, Kelly WG, Panning B: Chromatin remodeling in dosage compensation. Annu Rev Genet 2005, 39:615-651.

41. Fackelmayer FO: A stable proteinaceous structure in the territory of inactive $\times$ chromosomes. J Biol Chem 2005, 280(3):1720-1723.

42. Plath K, Fang J, Mlynarczyk-Evans SK, Cao R, Worringer KA, Wang H, de la Cruz CC, Otte AP, Panning B, Zhang Y: Role of histone H3 lysine 27 methylation in $\times$ inactivation. Science 2003, 300(5616):131-135.

43. Lucchesi JC, Manning JE: Gene dosage compensation in Drosophila melanogaster. Adv Genet 1987, 24:371-429.

44. Meyer BJ, Casson LP: Caenorhabditis elegans compensates for the difference in $\times$ chromosome dosage between the sexes by regulating transcript levels. Cell 1986, 47(6):871-881.

45. Schadt EE, Monks SA, Drake TA, Lusis AJ, Che N, Colinayo V, Ruff TG, Milligan SB, Lamb JR, Cavet G, et al: Genetics of gene expression surveyed in maize, mouse and man. Nature 2003, 422(6929):297-302.

46. Morley M, Molony CM, Weber TM, Devlin JL, Ewens KG, Spielman RS, Cheung VG: Genetic analysis of genome-wide variation in human gene expression. Nature 2004, 430(7001):743-747.

47. Kang HM, Ye C, Eskin E: Accurate discovery of expression quantitative trait loci under confounding from spurious and genuine regulatory hotspots. Genetics 2008, 180(4):1909-1925.

48. Breitling R, Li Y, Tesson BM, Fu J, Wu C, Wiltshire T, Gerrits A, Bystrykh LV, de Haan G, Su Al, et al: Genetical genomics: spotlight on QTL hotspots. PLoS Genet 2008, 4(10):e1000232.

49. Wu C, Delano DL, Mitro N, Su SV, Janes J, McClurg P, Batalov S, Welch GL, Zhang J, Orth AP, et al: Gene set enrichment in EQTL data identifies novel annotations and pathway regulators. PLoS Genet 2008, 4(5): e1000070.

50. Perez-Enciso M, Quevedo JR, Bahamonde A: Genetical genomics: use all data. BMC Genomics 2007, 8:69.

51. Bentwich I, Avniel A, Karov Y, Aharonov R, Gilad S, Barad O, Barzilai A, Einat $P$, Einav $U$, Meiri $E$, et al: Identification of hundreds of conserved and nonconserved human microRNAs. Nat Genet 2005, 37(7):766-770.

52. Laurent P, Schibler L, Vaiman A, Laubier J, Delcros C, Cosseddu G, Vaiman D, Cribiu EP, Yerle M: A 12 000-rad whole-genome radiation hybrid panel in sheep: application to the study of the ovine chromosome 18 region containing a QTL for scrapie susceptibility. Anim Genet 2007, 38(4):358-363.

53. Rink A, Eyer K, Roelofs B, Priest K, Sharkey-Brockmeier KJ, Lekhong S, Karajusuf EK, Bang J, Yerle M, Milan D, et al: Radiation hybrid map of the porcine genome comprising 2035 EST loci. Mamm Genome 2006, 17(8):878-885.

54. Womack JE, Johnson JS, Owens EK, Rexroad CE, Schlapfer J, Yang YP: A whole-genome radiation hybrid panel for bovine gene mapping. Mamm Genome 1997, 8(11):854-856.

55. Itoh T, Watanabe T, Ihara N, Mariani P, Beattie CW, Sugimoto Y, Takasuga A: A comprehensive radiation hybrid map of the bovine genome comprising 5593 loci. Genomics 2005, 85(4):413-424.

56. McCarthy LC, Bihoreau MT, Kiguwa SL, Browne J, Watanabe TK, Hishigaki H, Tsuji A, Kiel S, Webber C, Davis ME, et al: A whole-genome radiation hybrid panel and framework map of the rat genome. Mamm Genome 2000, 11(9):791-795.

57. Hitte C, Madeoy J, Kirkness EF, Priat C, Lorentzen TD, Senger F, Thomas D, Derrien T, Ramirez C, Scott C, et al: Facilitating genome navigation: survey sequencing and dense radiation-hybrid gene mapping. Nat Rev Genet 2005, 6(8):643-648.

58. Kent WJ, Baertsch R, Hinrichs A, Miller W, Haussler D: Evolution's cauldron: Duplication, deletion, and rearrangement in the mouse and human genomes. Proceedings of the National Academy of Sciences of the United States of America 2003, 100(20):11484-11489.

59. Schwartz S, Kent WJ, Smit A, Zhang Z, Baertsch R, Hardison RC, Haussler D, Miller W: Human Mouse Alignments with BLASTZ. Genome Research 2003, 13(1):103-107.

60. Hofacker IL, Fekete M, Flamm C, Huynen MA, Rauscher S, Stolorz PE, Stadler PF: Automatic detection of conserved RNA structure elements in complete RNA virus genomes. Nucl Acids Res 1998, 26(16):3825-3836.

61. Hofacker IL, Fontana W, Stadler PF, Bonhoeffer LS, Tacker M, Schuster P: Fast folding and comparison of RNA secondary structures. Monatshefte für Chemie/Chemical Monthly 1994, 125(2):167-188.
62. Stajich JE, Block D, Boulez K, Brenner SE, Chervitz SA, Dagdigian C, Fuellen G, Gilbert JGR, Korf I, Lapp H, et al: The Bioperl Toolkit: Perl Modules for the Life Sciences. Genome Research 2002, 12(10):1611-1618.

63. Kent WJ: BLAT - The BLAST-Like Alignment Tool. Genome Research 2002, 12(4):656-664

\section{doi:10.1186/1471-2164-12-562}

Cite this article as: Wang et al:: Effects of genome-wide copy number variation on expression in mammalian cells. BMC Genomics 2011 12:562.

\section{Submit your next manuscript to BioMed Central and take full advantage of:}

- Convenient online submission

- Thorough peer review

- No space constraints or color figure charges

- Immediate publication on acceptance

- Inclusion in PubMed, CAS, Scopus and Google Scholar

- Research which is freely available for redistribution 